\section{Changes in retinal nerve fibre layer, optic nerve head morphology, and visual field after acute primary angle closure}

CCA Sng', JSL See', CS Ngo'1, M Singh'1, Y-H Chan², MC Aquino', AM Tan', N Shabana' and PTK Chew ${ }^{1}$

\section{Abstract}

Aims/Purpose To determine and correlate the long-term changes in retinal nerve fibre layer (RNFL) thickness, optic nerve head (ONH) morphology, and visual fields after a single episode of acute primary angle closure (APAC).

Methods This was a cross-sectional comparative study of patients at National University Hospital (Singapore) from 2000 to 2006 after an episode of unilateral APAC. The peripapillary and macular RNFL were measured using Stratus optical coherence tomography (OCT) and ONH configuration was assessed using Heidelberg Retina Tomography (HRT)-III. Humphrey perimetry was also performed, and the presence of disc pallor was noted. APAC eyes were compared with fellow eyes as matched controls.

Results Twenty-five patients were assessed at a median of 33 months (range, 11-85 months) after APAC. OCT showed that there was a reduction in the peripapillary and outer macular RNFL thickness in APAC eyes compared with controls. Humphrey perimetry revealed significantly reduced mean deviation $(P=0.006)$ and increased pattern standard deviation $(P=0.045)$ in APAC eyes compared with controls. HRT-III showed no difference in mean rim area, rim volume, or cup-disc ratio between APAC eyes and controls. Disc pallor was present in nine APAC eyes $(36 \%)$ but was absent in fellow eyes $(P=0.002)$, and was associated with peripapillary RNFL thinning, visual field loss, and an increased interval between the onset of symptoms and normalization of intraocular pressure $(P=0.023)$.

Conclusion APAC results in peripapillary and outer macular RNFL loss, visual field defects, and optic disc pallor, even in cases in which the ONH configuration remains unchanged.

Eye (2011) 25, 619-625; doi:10.1038/eye.2011.31; published online 25 March 2011

Keywords: glaucoma; acute angle closure; imaging

\section{Introduction}

Acute primary angle closure (APAC) is an ophthalmic emergency as the sudden and excessive rise in intraocular pressure (IOP) is vision threatening if left untreated. Many studies report a poor long-term visual outcome after APAC, with high rates of blindness and glaucomatous optic neuropathy. ${ }^{1,2}$ Retinal nerve fibre layer (RNFL) loss, ${ }^{3-5}$ optic nerve head $(\mathrm{ONH})$ damage ${ }^{6,7}$ and visual field defects ${ }^{8-10}$ have been described following APAC. However, the severity of these sequelae is not well established, and little is known about the correlation between them.

Therefore, this study was performed to determine and correlate the long-term changes in peripapillary and macular RNFL thickness, $\mathrm{ONH}$ morphology, and visual fields after a single episode of APAC.

Materials and methods

\section{Patient selection}

This was a cross-sectional comparative study of patients who received treatment at National University Hospital in Singapore from 2000 to 2006 for an episode of unilateral APAC. These patients were identified from the hospital
${ }^{1}$ Department of Ophthalmology, National University Health System, Singapore

${ }^{2}$ Biostatistics Unit, National University of Singapore, Singapore

Correspondence: PTK Chew, Department of Ophthalmology, National University Health System, 1E Kent Ridge Road, Level 7, NUHS Tower Block, 119228 Singapore

Tel: + 656772 5317;

Fax: + 6567777161

E-mail: ophchewp@

nus.edu.sg

Received: 10 August 2010 Accepted in revised form: 7 January 2011

Published online: 25 March 2011

This work was presented as an oral presentation at the American Academy of Ophthalmology Meeting in 2009. 
computerized database, with the search terms of 'Acute Glaucoma' and 'Angle Closure' under the 'Diagnosis' category, and 'Peripheral Iridotomy' under the

'Treatment' category. The case notes of the list of patients obtained were reviewed, and APAC was defined by the following criteria: ${ }^{11}$

1. Presence of at least two of these symptoms: ocular or periocular pain, headache, nausea and/or vomiting, blurring of vision.

2. Presenting IOP of $>21 \mathrm{~mm} \mathrm{Hg}$ (as measured by Goldmann applanation tonometry).

3. The presence of at least three of these signs: conjunctival injection, corneal epithelial oedema, shallow anterior chamber and mid-dilated unreactive pupil.

4. Closed anterior chamber angles in four quadrants found on gonioscopic examination.

Patients with secondary angle closure, such as iris neovascularization, lens intumescence or subluxation, and uveitis were excluded. We also excluded patients who had evidence of primary angle closure glaucoma (PACG) in the APAC eye at presentation. We defined PACG as any one or both findings below: ${ }^{11}$

1. The presence of glaucomatous optic neuropathy, with loss of neuroretinal rim tissue and a vertical cup-disc ratio (CDR) of $\geq 0.6$ and/or

2. Significant visual field defects on static automated white-on-white threshold perimetry (detailed further on in the section).

Demographic data were recorded for every patient. The presenting IOP, the time interval between onset of symptoms and presentation, the interval between onset of symptoms and normalization of IOP (time interval between onset of symptoms and IOP $\leq 21 \mathrm{~mm} \mathrm{Hg}$ ), other ocular pathologies, and the presence of any ocular surgeries were documented. Approval for this study was obtained from the institutional ethical board. Patient consent was obtained and the study was conducted in accordance with the Declaration of Helsinki.

Subjects who fulfilled the above inclusion criteria were identified from the clinic database and were contacted by phone. They were invited to attend a clinic examination, which included an ophthalmic examination, RNFL imaging using time-domain optical coherence tomography (OCT), confocal scanning laser ophthalmoscopy (CSLO) of the ONH, and automated perimetry. APAC eyes were compared with the fellow eyes as controls. Study assessments were performed at a mean of 39.6 months (range, 11-85 months) after APAC.

\section{Ophthalmic examination}

Detailed examination of both eyes was performed on all participants by a single ophthalmologist, including slit-lamp examination of the anterior segment, Goldmann applanation tonometry, and dilated fundal examination. The best-corrected visual acuity (BCVA) was documented. The presence of a cataract, defined as an opacification of the crystalline lens, was noted, and the severity of the cataract was graded with Lens Opacity Classification System III (LOCS III). ${ }^{12}$

\section{RNFL imaging}

RNFL imaging was performed by experienced full-time operators in both eyes using time-domain OCT (Stratus OCT 3000, Carl Zeiss, Meditec Inc., Dublin, CA, USA). Peripapillary and macular RNFL measurements were obtained using the fast RNFL thickness (3.4) protocol. For the peripapillary RNFL, the average thickness $\left(360^{\circ}\right.$ measurement), the mean thickness of the superior and inferior quadrants, and the difference between the maximum and minimum RNFL thickness (max-min) were analysed. For the macular RNFL, the mean thickness of the fovea, as well as the superior, inferior, nasal, and temporal quadrants of the inner and outer macula were analysed. Only images with signal strength of $\geq 5$ (maximum 10) were included. The signal strength of OCT images was $>6$ in 20 patients, but $<6$ in the attack or fellow eye of five patients. Exclusion of OCT images with signal strength $<6$ from the analysis yielded similar results. Mean RNFL thickness and RNFL height variation contour measured using Heidelberg Retinal Tomography (HRT)-III (Heidelberg Engineering Inc., Vista, CA, USA) were also recorded.

\section{Assessment of the ONH morphology}

All subjects underwent CSLO of the ONH using HRT-III (Heidelberg Engineering Inc.). The following indices were documented: linear CDR, rim area, rim volume, disc size, Moorfields Regression Classification, and the R Burk (RB) and FS Mikelberg (FSM) linear discriminant functions (LDFs). The presence of optic disc pallor was noted clinically by a single ophthalmologist, with a slit beam of light and then comparing the appearance with the contralateral optic disc. Using a narrow beam of light allows more accurate colour appreciation by limiting 'colour leak' from the peripapillary choroids to the adjacent optic disc, giving a falsely pink appearance to pale disc tissue. ${ }^{13}$ Optic disc stereo photography was also performed, and the presence of disc pallor on stereo photographs was noted independently by two masked observers who were only given information on the 
phakic status of each eye. Disc pallor was excluded in subjects who underwent unilateral cataract extraction, and had comparatively paler optic discs in the pseudophakic eyes, as this was likely secondary to false appreciation of optic disc colour produced by alterations in the optical media of the eye. In cases of disagreement between clinical examination and the assessment of disc colour on stereo photographs, the disc colour on stereo photographs would be used in the subsequent analysis. A masked observer (PTKC), who is an experienced ophthalmologist with more than 20 years of experience in the field of glaucoma, determined whether each optic disc obeyed the ISNT rule on stereo photographs.

\section{Automated perimetry}

Subjects underwent automated static white-on-white perimetry (program 24-2, SITA standard, Humphrey Instruments, Dublin, CA, USA). The criteria used for reliability were fixation losses $<20 \%$, false positive $<33 \%$, and false negative $<33 \%$. Subjects with unreliable visual fields underwent repeat tests, up to a maximum of three attempts, after which they would be excluded from the study. The global indices including mean deviation (MD) and corrected pattern standard deviation (PSD) of the visual fields were documented. A significant visual field defect was defined as follows:

1. Glaucoma Hemifield test outside normal limits and

2. $\geq 3$ contiguous points on the pattern deviation plot depressed at $P<5 \%$ level.

\section{Statistical analysis}

Statistical analysis was performed using the system of Statistical Products and Services Solution (SPSS version 14.0, Chicago, IL, USA). The differences in RNFL thickness, $\mathrm{ONH}$ configuration, and visual field between the APAC eyes and the fellow eyes as matched controls were compared using the Wilcoxon Signed Rank test, and the development of disc pallor in APAC eyes was analysed using the McNemar's test with relative risks presented. The McNemar's test was also used to compare the differences in Moorfields Regression Classification, the ISNT rule, and the RB and FSM LDFs between APAC eyes and fellow eyes. The MannWhitney $U$ test was used to compare the differences in RNFL thickness, ONH configuration, and visual field between APAC eyes that developed disc pallor and those that did not; and also to compare the differences in these parameters between APAC eyes that had significant visual field defects and those that did not. The Fisher's exact test was used to determine the association between APAC eyes with disc pallor and the presence of significant visual field defects with odds ratios presented. Inter-observer agreement for the assessment of disc colour on stereo photographs was determined by $\kappa$ statistics. Statistical significance was set at $P<0.05$.

\section{Results}

Thirty-one subjects who fulfilled the above inclusion criteria were identified from the clinic database. Two subjects could not be contacted due to an invalid telephone number and four contacted subjects declined participation, leaving 25 participants $(80.6 \%)$ who attended the clinic examination. Study assessments were performed at a median of 33 months (range, 11-85 months) after APAC. The demographics of the subjects included in this study and the features of the APAC attack are summarized in Table 1.

In all, 22 of the 25 participants $(88.0 \%)$ had cataract in the attack eye at the time of presentation with APAC, but no other co-existing ocular pathologies. The BCVA for all APAC and fellow eyes was $\geq 6 / 12$ and all phakic eyes (both APAC and fellow eyes) had mild nuclear sclerosis cataracts of LOCS III grading NI at the time of

Table 1 Characteristics of study participants

\begin{tabular}{lc}
\hline Age (years) at the time of presentation & \\
Mean (SD) & $58.4(8.3)$ \\
Range & $39-72$ \\
Gender & \\
$\quad$ Male & $7(28.0 \%)$ \\
Female & $18(72.0 \%)$ \\
& \\
Race & \\
Chinese & $22(88.0 \%)$ \\
Malay & $3(12.0 \%)$ \\
Follow-up (months) & \\
Median (SD) & \\
Range & $33(24.6)$ \\
\end{tabular}

Interval (hours) between the onset of APAC symptoms and presentation

Mean (SD) 27.7 (22.5)

Range

$5-96$

Interval (hours) between the onset of APAC symptoms and normalization of IOP

Mean (SD)

Range

$43.4(27.7)$

3-101

Interval (hours) between presentation and normalization of IOP Mean (SD)

$17.1(19.1)$

Range

$1-80$

Presenting IOP $(\mathrm{mm} \mathrm{Hg})$

Mean (SD)

$57.1(12.0)$

Range 37-88

Abbreviations: APAC, acute primary angle closure; IOP, intraocular pressure. 
assessment. Combined trabeculectomy and cataract extraction was performed on five APAC eyes $(20.0 \%)$ at a mean of 8.1 months (range, $0.25-29$ months) after the attack. One APAC eye (4.0\%) underwent combined cataract extraction with insertion of glaucoma drainage device 16 months after the attack. Cataract extraction alone was performed on six APAC eyes (25.0\%) at a mean of 6.3 months (range, $0.02-38$ months) after the attack. One APAC eye required IOP-lowering medication but had no subsequent ocular surgery.

Laser peripheral iridotomy was performed on all the fellow eyes within 1 month of the APAC attack, and five fellow eyes had undergone cataract extraction at the time of the study assessment. All records of IOP in the fellow eyes were $\leq 21 \mathrm{~mm} \mathrm{Hg}$ following APAC. The IOP was also $\leq 21 \mathrm{~mm} \mathrm{Hg}$ at the time of the study assessment. None of the fellow eyes required glaucoma filtration surgery or IOP-lowering medication.

\section{Comparison between APAC eyes and fellow eyes}

The comparison of RNFL thickness, ONH configuration, and visual field parameters between APAC eyes and fellow eyes are summarized in Table 2.
In our study, five subjects were bilaterally pseudophakic, 13 were bilaterally phakic, and seven were pseudophakic in one eye and phakic in the other eye. Nine APAC eyes (36\%) had disc pallor detectable clinically and on stereo photographs, compared with none $(0 \%)$ of the fellow eyes $(P=0.002$, relative risk 1.6 , $95 \%$ confidence interval 1.2-2.1). The agreement between the two observers assessing disc colour on the stereo photographs was $100 \%$, and there was also a $100 \%$ agreement with the clinical assessment of disc colour. Among the seven subjects whose phakic status differed in each eye, four subjects had symmetrically pink discs in both eyes, as the phakic eye had very mild cataracts (LOCS III grading NI). In the remaining three subjects, the optic disc appeared slightly paler on stereo photographs in the pseudophakic eye than in the phakic eye. However, with the knowledge of the phakic status in each eye, both masked observers independently decided that the optic disc in the pseudophakic eye was pink. None of the patients suffered from other ocular pathologies that could result in optic disc pallor, such as toxic or nutritional optic neuropathy, previous optic nerve inflammation, compressive optic neuropathy, chronic papilloedema, retinal dystrophy, and previous

Table 2 Comparison of retinal nerve fibre layer thickness, optic nerve head configuration, and visual field parameters between APAC eyes and fellow eyes

\begin{tabular}{|c|c|c|c|}
\hline Parameter & $\begin{array}{c}A P A C \text { eyes } \\
(\text { mean } \pm S D)\end{array}$ & $\begin{array}{l}\text { Fellow eyes } \\
(\text { mean } \pm S D)\end{array}$ & P-value \\
\hline \multicolumn{4}{|c|}{ Mean RNFL thickness assessed by stratus OCT ( $\mu m)$} \\
\hline \multicolumn{4}{|l|}{ Peripapillary RNFL } \\
\hline Average thickness & $87.1 \pm 20.8$ & $107.5 \pm 14.2$ & $<0.001^{*}$ \\
\hline Superior & $107.6 \pm 29.0$ & $135.4 \pm 24.6$ & $<0.001^{*}$ \\
\hline Inferior & $106.4 \pm 33.7$ & $139.7 \pm 19.9$ & $<0.001^{*}$ \\
\hline Max-min & $107.6 \pm 35.7$ & $134.8 \pm 21.2$ & $0.001^{*}$ \\
\hline \multicolumn{4}{|l|}{ Macular RNFL } \\
\hline Inner superior & $247.2 \pm 19.5$ & $249.6 \pm 29.9$ & 0.696 \\
\hline Inner inferior & $246.9 \pm 19.1$ & $254.4 \pm 23.2$ & 0.085 \\
\hline Inner temporal & $235.1 \pm 16.3$ & $241.8 \pm 26.5$ & 0.128 \\
\hline Inner nasal & $247.0 \pm 22.0$ & $251.9 \pm 23.1$ & 0.278 \\
\hline Outer superior & $223.0 \pm 18.6$ & $229.5 \pm 23.8$ & $0.030^{*}$ \\
\hline Outer inferior & $210.2 \pm 17.3$ & $224.2 \pm 16.4$ & $<0.001^{*}$ \\
\hline Outer temporal & $203.2 \pm 16.2$ & $214.0 \pm 16.9$ & $0.001^{*}$ \\
\hline Outer nasal & $238.0 \pm 18.6$ & $244.9 \pm 16.4$ & $0.030^{*}$ \\
\hline Fovea & $176.3 \pm 22.7$ & $171.6 \pm 28.0$ & 0.339 \\
\hline \multicolumn{4}{|c|}{ ONH configuration assessed by HRT-III } \\
\hline $\operatorname{Rim}$ area $\left(\mathrm{mm}^{2}\right)$ & $1.62 \pm 0.37$ & $1.62 \pm 0.31$ & 0.55 \\
\hline Rim volume $\left(\mathrm{mm}^{3}\right)$ & $0.41 \pm 0.16$ & $0.48 \pm 0.18$ & 0.08 \\
\hline Linear CDR & $0.44 \pm 0.20$ & $0.39 \pm 0.20$ & 0.80 \\
\hline Disc size $(\mathrm{mm})$ & $2.14 \pm 0.39$ & $2.05 \pm 0.48$ & 0.07 \\
\hline \multicolumn{4}{|c|}{ Visual field assessed by Humphrey perimetry } \\
\hline MD & $-4.79 \pm 3.29$ & $-2.75 \pm 2.02$ & $0.006^{*}$ \\
\hline PSD & $3.15 \pm 1.89$ & $2.45 \pm 1.63$ & $0.045^{*}$ \\
\hline
\end{tabular}

Abbreviations: APAC, acute primary angle closure; CDR, cup-disc ratio; HRT, Heidelberg Retina Tomography; MD, mean deviation; OCT, optical coherence tomography; ONH, optic nerve head; PSD, pattern standard deviation; RNFL, retinal nerve fibre layer. $* P<0.05$. 
retinal laser. The ISNT rule was not maintained in one APAC eye and four fellow eyes. Comparing the APAC and fellow eyes, there was no significant difference in the proportion of eyes that did not obey the ISNT rule $(P=0.38)$. The FSM LDF classified three APAC eyes and six fellow eyes as diseased $(P=0.62)$, while the RB LDF classified none of the APAC eyes and two fellow eyes as diseased $(P=0.48)$. Moorfields Regression Classification was abnormal for four APAC eyes and three fellow eyes, and this difference was not statistically significant $(P=1.0)$. Mean RNFL thickness measured using HRT-III was not significantly different between APAC and fellow eyes $(P=0.103)$, but the height variation contour was significantly smaller for APAC eyes compared with fellow eyes (0.39 vs 0.44, $P=0.047)$.

\section{Comparison between APAC eyes with pale discs and pink discs}

Table 3 summarizes the parameters that were significantly different between APAC eyes with pale discs and APAC eyes with pink discs. A higher proportion of APAC eyes with disc pallor had significant visual field defects compared with those with pink discs (66.7 vs 12.5\%, $P=0.010$, odds ratio 14.0). The presence of disc pallor was significantly associated with an increased interval between the onset of symptoms and normalization of IOP compared with those with pink discs $(59.3 \pm 19.9$ vs $33.7 \pm 28.0 \mathrm{~h}, P=0.023)$. Comparing APAC eyes with pale discs to APAC eyes with pink discs, there was no significant difference in the presenting IOP, presenting CDR, outer and inner macular RNFL thickness, rim area, rim volume, linear CDR, and disc size.

\section{Comparison between APAC eyes with and without significant visual field defects}

Eight APAC eyes (32.0\%) developed PACG with significant visual field defects. Comparing APAC eyes that developed significant visual field defects with those that did not showed that the presence of significant visual field defects was associated with reduced average (74.6 \pm 21.3 vs $92.9 \pm 18.2 \mathrm{~mm}, P=0.048$ ), inferior (85.5 \pm 32.0 vs $116.3 \pm 30.5 \mathrm{~mm}, P=0.039)$ and max-min $(86.1 \pm 36.2$ vs $117.7 \pm 31.6 \mathrm{~mm}, P=0.041)$ peripapillary RNFL thickness. Superior peripapillary RNFL thickness, outer and inner macular RNFL thickness, rim area, rim volume, linear CDR, and disc size were not significantly different between APAC eyes with significant visual field defects and those without.

\section{Discussion}

A single episode of APAC was associated with RNFL thinning, visual field defects, and pale discs in this group of patients who were assessed at least 11 months after APAC. However, ONH configuration assessed by HRT-III was not significantly altered. Disc pallor was significantly associated with visual field defects, peripapillary RNFL thinning, and an increased interval between the onset of symptoms and normalization of IOP.

Our results suggest that disc pallor following APAC was associated with RNFL loss, visual field defects, and an increased interval between the onset of symptoms and normalization of IOP, which have not been previously reported. However, these results are preliminary and require validation in further studies. In our study, optic disc configuration measured by HRT-III was not significantly altered after APAC of short duration (mean interval between the onset of symptoms and normalization of IOP $=43.4 \mathrm{~h}$ ). This is unlike the findings of Shen et $a l^{7}$ who described an increase in the mean $\mathrm{CDR}$ and a decrease in the mean neuroretinal rim area as assessed by stereo photographs from week 2 to week 16 after APAC. However, these longitudinal changes in optic disc morphology may be due to the resolution of disc swelling, which has been described in association

Table 3 Comparison between APAC eyes with pale discs and APAC eyes with pink discs

\begin{tabular}{lccc}
\hline Parameter & $\begin{array}{c}\text { Pale disc } \\
(\text { mean } \pm S D)\end{array}$ & $\begin{array}{c}\text { Pink disc } \\
\text { (mean } \pm S D)\end{array}$ & P-value \\
\hline Mean peripapillary RNFL thickness & assessed by stratus OCT $(\mu \mathrm{m})$ & & $0.002^{*}$ \\
$\quad$ Average thickness & $70.8 \pm 15.6$ & $96.2 \pm 17.7$ & $0.004^{*}$ \\
Superior & $85.6 \pm 19.7$ & $120.0 \pm 26.1$ & $0.002^{*}$ \\
Inferior & $80.6 \pm 25.3$ & $121.0 \pm 29.0$ & $0.001^{*}$ \\
Max-min & $107.6 \pm 35.7$ & $134.8 \pm 21.2$ & $0.001^{*}$ \\
Visual field assessed by Humphrey perimetry & & & $0.007^{*}$ \\
MD & $-7.63 \pm 3.15$ & $-3.19 \pm 2.12$ & $2.53 \pm 1.71$ \\
PSD & $4.25 \pm 1.77$ & & \\
\hline
\end{tabular}

Abbreviations: APAC, acute primary angle closure; MD, mean deviation; OCT, optical coherence tomography; PSD, pattern standard deviation; RNFL, retinal nerve fibre layer.

${ }^{*} P<0.05$. 
with APAC, ${ }^{14}$ rather than an actual loss of rim area. Previous studies have shown that abnormally shaped optic nerve rim width (with alteration of the ISNT rule) was more significantly associated with chronic PACG than APAC, and hypothesized that this was related to the duration of increased IOP. ${ }^{9}$ Optic disc pallor has been described in 20-39\% of eyes following APAC, ${ }^{1,15}$ though the visual consequences of this are unclear. Our results show that pale discs are associated with visual field defects. There are a few possible reasons for the development of disc pallor without a change in optic disc configuration. First, the axonal loss resulting from the short duration of raised IOP might not be extensive enough to be detectable on HRT-III. Studies on primates have shown that an increase in the CDR is seen only when IOP is elevated for more than 1 week. ${ }^{16}$ Second, it might be possible that gliolization has replaced any lost tissue in the optic disc. ${ }^{17,18}$ The compression of vessels in the prelaminar region when IOP is increased could also lead to ischaemia and pallor of the optic disc without a change in the optic disc configuration, similar to the changes following anterior ischaemic optic neuropathy. ${ }^{19}$ In contrast, a longer duration of raised IOP during APAC may result in the formation of peripheral anterior synechiae, subsequent IOP elevation despite a patent peripheral iridotomy, and optic disc cupping. ${ }^{1,2,6,9}$

Our finding that peripapillary RNFL loss following APAC was associated with pale discs and visual field loss has not been previously reported. Previous studies have shown that an episode of APAC is associated with RNFL loss, detectable by both scanning laser polarimetry and $\mathrm{OCT}_{,}^{3-5}$ but the functional consequence of this is unclear. Our results also provide new data that APAC results in a decrease in outer macular volume, though this was not associated with significant visual field defects or optic disc pallor. Earlier studies indicate that macular thickness is decreased in both paediatric ${ }^{20,21}$ and adult glaucoma ${ }^{22-25}$ patients. We found that macular RNFL loss in APAC eyes was mild and $<10 \%$ compared with fellow eyes, hence was unlikely to be extensive enough to cause visual field defects on standard white-on-white perimetry.

The incidence of visual field loss was $32 \%$ in our study, which is similar to that reported by Aung et al. ${ }^{8}$ However, an earlier study, also conducted in Singapore, showed that up to $60 \%$ of patients had visual field loss that was moderate or worse. ${ }^{9}$ This discrepancy is likely due to the shorter duration of raised IOP in APAC eyes over time, as patients present earlier and the treatment of APAC is more aggressive. ${ }^{11}$ Early cataract extraction further prevents the development of PACG. ${ }^{11}$ We have also excluded those with evidence of PACG at presentation, while such patients were included in previous studies. ${ }^{1,2}$ In our study, patients presented within 4 days of the onset of symptoms, hence the visual field loss seen in APAC eyes was mild, with an average MD of -4.79 . However, a significant difference between APAC and fellow eyes was detected, with APAC eyes having a more negative MD and a more positive PSD, despite a higher proportion of pseudophakia in APAC eyes compared with fellow eyes. We provide new data that the presence of significant visual field defects following APAC was associated with RNFL loss and pale discs.

Among the main strengths of this study were that visual field changes following APAC with optic disc corresponded on the average with the RNFL findings in the groups of patients with and without pale discs (Table 3), the objective measurement of optic disc configuration with HRT-III, and the analysis of macular RNFL after APAC. These findings have not been previously reported. As none of the fellow eyes developed raised IOP following APAC, or required IOPlowering medication or glaucoma filtration surgery, they were appropriate controls. However, these results should be interpreted in view of the limitations inherent in this study, in particular the small final sample size. The main reason for this was that a substantial proportion of patients who presented to the National University Hospital with APAC between 2000 and 2006 were not entered into the hospital database with the appropriate 'Diagnosis' or 'Treatment' codes, hence could not be identified. Nevertheless, the response rate was high for those we were able to locate and contact, and there was no difference in the clinical characteristics of those entered into the clinical database as compared with the overall group who presented with APAC. Despite the small numbers, most of the $P$-values obtained during analysis were very low, indicating robust statistical significance. Second, this was a cross-sectional study, hence the follow-up interval varied greatly. However, all subjects were examined $>11$ months after the resolution of APAC, and a previous study has indicated that most patients progress to PACG within 6 months of APAC. ${ }^{2}$ Though data on RNFL, ONH morphology, and visual field were collected prospectively, details of the APAC episode were obtained retrospectively. Third, the signal strength for OCT images was $<6$ in five patients. However, these images were still included in the final analysis as the sample size was small, and exclusion of these patients in the analysis of RNFL thickness yielded similar results.

This study has shown that a single episode of APAC was associated with peripapillary RNFL thinning, mild visual field defects, and optic disc pallor. The presence of pale discs following APAC was associated with peripapillary RNFL loss, visual field defects, and an increased interval between the onset of symptoms and normalization of IOP. 
Summary

\section{What was known before}

- Retinal nerve fibre layer (RNFL) loss, optic nerve head $(\mathrm{ONH})$ damage, and visual field defects have been described following APAC. However, the severity of these sequelae is not well established, and little is known about the correlation between them.

\section{What this study adds}

- This study has shown that a single episode of APAC was associated with peripapillary RNFL thinning, mild visual field defects, and optic disc pallor. The presence of pale discs following APAC was associated with peripapillary RNFL loss, visual field defects, and an increased interval between the onset of symptoms and normalization of IOP.

\section{Conflict of interest}

The authors declare no conflict of interest

\section{Acknowledgements}

We thank Dr Balwantray Chauhan for his help in the preparation of the manuscript.

\section{References}

1 Aung T, Friedman D, Chew P, Ang LP, Gazzard G, Lai YF et al. Long-term outcomes in Asians after acute primary angle closure. Ophthalmology 2004; 111(8): 1464-1469.

2 Aung T, Ang L, Chan S, Chew P. Acute primary angleclosure: long-term intraocular pressure outcome in Asian eyes. Am J Ophthalmol 2001; 131(1): 7-12.

3 Tsai J, Chang H. Scanning laser polarimetry in patients with acute angle-closure glaucoma. Eye 2004; 18(1): 9-14.

4 Fang A, Qu J, Li L, Ji B. Measurement of retinal nerve fiber layer in primary acute angle closure glaucoma by optical coherence tomography. J Glaucoma 2007; 16(2): 178-184.

5 Aung T, Husain R, Gazzard G, Chan YH, Devereux JG, Hoh ST et al. Changes in retinal nerve fiber layer thickness after acute primary angle closure. Ophthalmology 2004; 111(8): 1475-1479.

6 Uhm K, Lee J, Sung H. Comparison of glaucomatous optic nerve damage in primary angle-closure glaucoma with and without acute attack. Korean J Ophthalmol 2005; 19(3): 201-207.

7 Shen S, Baskaran M, Fong A, Chan YH, Lim LS, Husain R et al. Changes in the optic disc after acute primary angle closure. Ophthalmology 2006; 113(6): 924-929.

8 Aung T, Looi A, Chew P. The visual field following acute primary angle closure. Acta Ophthalmol Scand 2001; 79(3): 298-300.

9 Ang L, Aung T, Chua W, Yip L, Chew P. Visual field loss from primary angle-closure glaucoma: a comparative study of symptomatic and asymptomatic disease. Ophthalmology 2004; 111(9): 1636-1640.
10 Bonomi L, Marraffa M, Marchini G, Canali N. Perimetric defects after a single acute angle-closure glaucoma attack. Graefes Arch Clin Exp Ophthalmol 1999; 237(11): 908-914.

11 Tan A, Loon S, Chew P. Outcomes following acute primary angle closure in an Asian population. Clin Exp Ophthalmol 2009; 37(5): 467-472.

12 Chylack Jr LT, Wolfe J, Singer D, Leske MC, Bullimore MA, Bailey IL et al. The Lens Opacity Classification System III. The Longitudinal Study of Cataract Study Group. Arch Ophthalmol 1993; 111: 831-836.

13 Hitchings R. The optic disc in glaucoma, III: diffuse optic disc pallor with raised intraocular pressure. $\mathrm{Br} \mathrm{J} \mathrm{Ophthalmol}$ 1978; 62(10): 670-675.

14 Yip L, Yong V, Hoh S, Wong H. Optical coherence tomography of optic disc swelling in acute primary angle-closure glaucoma. Arch Ophthalmol 2005; 123(4): 567-569.

15 Douglas G, Drance S, Schulzer M. The visual field and nerve head in angle-closure glaucoma. A comparison of the effects of acute and chronic angle closure. Arch Ophthalmol 1975; 93(6): 409-411.

16 Quigley H, Addicks E. Chronic experimental glaucoma in primates. II. Effect of extended intraocular pressure elevation on optic nerve head and axonal transport. Invest Ophthalmol Vis Sci 1980; 19(2): 137-152.

17 Johnson E, Morrison J. Friend or foe? Resolving the impact of glial responses in glaucoma. J Glaucoma 2009; 18(5): 341-353.

18 Quigley H, Guy J, Anderson D. Blockade of rapid axonal transport. Effect of intraocular pressure elevation in primate optic nerve. Arch Ophthalmol 1979; 97(3): 525-531.

19 Hayreh S. Blood supply of the optic nerve head and its role in optic atrophy, glaucoma and oedema of the optic disc. Br J Ophthalmol 1969; 53(11): 721-748.

20 El-Dairi M, Holgado S, Asrani S, Enyedi L, Freedman S. Correlation between optical coherence tomography and glaucomatous optic nerve head damage in children. Br J Ophthalmol 2009; 93(10): 1325-1330.

21 Hess D, Asrani S, MG; B, Enyedi L, Stinnett S, Freedman S. Macular and retinal nerve fiber layer analysis of normal and glaucomatous eyes in children using optical coherence tomography. Am J Ophthalmol 2005; 139(2): 509-517.

22 Ozturk F, Yavas G, Kusbeci T, Ermis S. A comparison among Humphrey field analyzer, Microperimetry, and Heidelberg Retina Tomograph in the evaluation of macula in primary open angle glaucoma. J Glaucoma 2008; 17(2): 118-121.

23 Ojima T, Tanabe T, Hangai M, Yu S, Morishita S, Yoshimura N. Measurement of retinal nerve fiber layer thickness and macular volume for glaucoma detection using optical coherence tomography. Jpn J Ophthalmol 2007; 51(3): 197-203.

24 Greenfield D, Bagga H, Knighton R. Macular thickness changes in glaucomatous optic neuropathy detected using optical coherence tomography. Arch Ophthalmol 2003; 121(1): 41-46.

25 Leung C, Chan W, Yung W, Ng AC, Woo J, Tsang MK et al. Comparison of macular and peripapillary measurements for the detection of glaucoma: an optical coherence tomography study. Ophthalmology 2005; 112(3): 391-400. 